Bio - grafía. Escritos sobre la Biología y su Enseñanza. ISSN 2027-1034

Edición Extraordinaria. p.p. 1149 - 1156

Memorias del IX Encuentro Nacional de Experiencias en Enseñanza de la Biología y la

Educación Ambiental. IV Congreso Nacional de Investigación en Enseñanza de la

Biología.

\title{
FUNCIONES DE LAS TIC EN LA ENSEÑANZA: UNA REVISIÓN DOCUMENT AL ${ }^{1}$ FUNCTIONS OF ICT IN TEACHING BIOLOGY. ADOCUMENT ARY REVIEWS
}

\author{
Héctor Alexander Afanador Castañeda ${ }^{2}$ \\ Edgar Orlay Valbuena Ussa ${ }^{3}$
}

\section{RESUMEN}

Se presentan resultados de la revisión documental sobre las funciones de las TIC en práctica de enseñanza mediada por TIC. Esta revisión interpretativa recurrió al análisis del contenido de 57 artículos científicos entre el periodo 2005 - 2015, pertenecientes a investigaciones de Nigeria, Kenia, Botsuana, Marruecos, Rumania, Eslovaquia, Eslovenia, República Checa, España, Francia, Turquía, India, México, Cuba, Colombia, Brasil, Argentina, Australia, Indonesia y Estados Unidos, entre otros, estableciendo la categoría descriptiva uso de TIC y su respectiva subcategoría (funciones de las TIC). Los datos de la bibliometría establecieron que existen pocos artículos de investigación en la enseñanza mediada con TIC, la mayor contribución fue de Suramérica y Europa. Y puede concluirse que las funciones de las TIC asignadas por los profesores yacen de la misma cotidianidad que luego son transferidas y adaptadas a la enseñanza.

PALABRAS CLAVES: enseñanza, funciones de las TIC, TIC.

\section{ABSTRACT}

Results of the documentary review on the functions of ICT in ICT-mediated teaching practice. This interpretive review was based on the analysis of the content of 57 scientific articles between 2005 and 2015, belonging to research from Nigeria, Kenya, Botswana, Morocco, Romania, Slovakia, Slovenia, Spain, France, Turkey, India, Mexico, Cuba, Colombia, Brazil, Argentina, Australia, Indonesia and the United States, among others, establishing the descriptive category use of ICT and its respective subcategory (functions of ICT). Data from the bibliometrics established that there are few research articles in the teaching mediated with ICT, the greatest contribution was from South America and

\footnotetext{
${ }^{1}$ Los resultados presentados forman parte de la investigación "Configuración de la práctica de enseñanza a través de TIC de profesores de Biología de la Secretaría de Educación Distrital, Bogotá", suscrita en el grupo de investigación Conocimiento Profesional del Profesor de la Universidad Pedagógica Nacional.

${ }^{2}$ Doctorando del Interinstitucional de Educación Universidad Pedagógica Nacional. Correo haacster@gmail.com.

${ }^{3}$ Docente del Doctorado Interinstitucional de Educación, Universidad Pedagógica Nacional, sede Bogotá. Correo edgarorlay@hotmail.com
} 
Bio - grafía. Escritos sobre la Biología y su Enseñanza. ISSN 2027-1034

Edición Extraordinaria. p.p. 1149 - 1156

Memorias del IX Encuentro Nacional de Experiencias en Enseñanza de la Biología y la

Educación Ambiental. IV Congreso Nacional de Investigación en Enseñanza de la

Biología.

Europe. And it can be concluded that the functions of ICT assigned by teachers lie in the same everyday life that they are then transferred and adapted to teaching.

KEY WORDS: teaching, functions of ICT, ICT.

\section{INTRODUCCIÓN}

La UNESCO (2013), considera que la existencia de TIC se ha convertido en una oportunidad y a la vez un desafío. Con ello surge la tarea de encontrar para ellas un sentido y uso. Además, la introducción de estas herramientas tecnológicas en la enseñanza genera cambios particulares en los usuarios, es decir la frecuencia de uso o utilización obedece a las necesidades de cada sujeto en aula (Escorcia \& James 2015), por lo tanto, adquiere una función y significado. Al respecto Almerich, Suárez, Jornet y Orellana, (2010), consideran la existencia de dos usos diferenciados en los recursos tecnológicos (nivel personal y profesional, y nivel de enseñanza y aprendizaje). En este sentido es importante preguntarse ¿En qué medida la presencia de las TIC, es esencial dentro de la práctica de enseñanza? o ¿cómo se explica que los recursos tecnológicos específicos desarrollan una función específica en la práctica de enseñanza?

Al interpretar a Bautista, Nafría, \& Salazar (2006) y Sancho (1995) sobre la funcionalidad de las TIC en la práctica de enseñanza, puede explicarse que es necesario establecer la relación entre el equipamiento técnico y la utilización de los recursos tecnológicos, e incluir la función de enseñanza acorde con su condición propia tecnológica (función primaria), con el propósito de facilitar a los profesores el desarrollo adecuado de actividades de enseñanza. En este sentido, la enseñanza a través de TIC, responde a maneras diversas de utilizar TIC para satisfacer una variedad de objetivos de enseñanza y de aprendizaje, que permita el desarrollo del aprendizaje en un contexto particular, de tal modo que los artefactos tendrán que convertirse en una parte integral de la estrategia didáctica (Niederhauser y Stoddart, 2001), es decir adquirir funcionalidad en la enseñanza y el aprendizaje. Por lo tanto, el objetivo que se pretende desarrollar es determinar qué clase de funciones tienen las TIC en la práctica de enseñanza.

\section{DISEÑO METODOLÓGICO}

La presente revisión documental bibliográfica empleó el análisis de contenido para identificar e interpretar unidades de análisis relacionadas con las funciones de las TIC en la enseñanza de la Biología. A continuación, la estrategia de investigación se presenta en dos etapas.

Etapa uno, bibliometría de la revisión documentada

Esta etapa tuvo como objetivo identificar la vigencia y relevancia de la revisión documental en torno a las funciones de las TIC en la enseñanza, específicamente de la Biología, dentro del periodo cronológico 2005 y 2015 puesto que corresponde al periodo 
Bio - grafía. Escritos sobre la Biología y su Enseñanza. ISSN 2027-1034

Edición Extraordinaria. p.p. 1149 - 1156

Memorias del IX Encuentro Nacional de Experiencias en Enseñanza de la Biología y la

Educación Ambiental. IV Congreso Nacional de Investigación en Enseñanza de la

Biología.

con mayor fuerza de desarrollo de políticas públicas colombianas en educación sobre la incorporación de las TIC en la enseñanza. Para el éxito de la productividad se adoptó la búsqueda y selección de información a partir de plataformas electrónicas (google académico, dialnet.com, ScieELO.org, sciencedirect.com, oei.es, ojs.pensamultimedia, eduteka.org y ries.universia.net, entre otras), permitiendo la identificación y evaluación de fuentes potenciales de información y al acceso a las fuentes de información seleccionadas.

Etapa dos, análisis del contenido de la revisión documental

Se asumió la unidad de análisis con el objetivo de detectar la presencia de las cualidades (funciones de las TIC) en el contenido de los artículos científicos y para formular como una subcategoría de eje principal uso de TIC que facilite la comprensión de dicha categoría. Con ello a la vez se contribuye con la productividad diacrónica y de contenido, al cual se refieren Anta y Pérez (2004). Y sobre el cual se realiza la discusión interpretativa.

\section{RESULT ADOS BIBLIOMÉTRICOS}

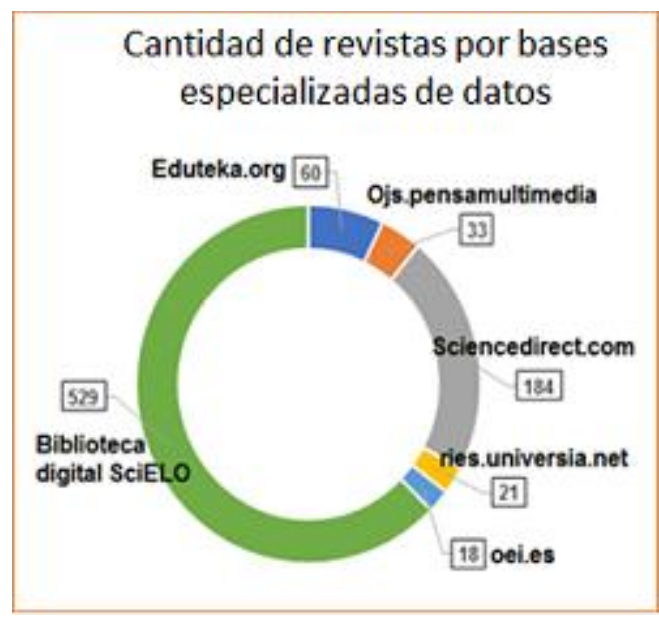

Gráfica 1. Cantidad de artículos científicos por bases especializadas de datos.

\section{Origen de las Investigaciones}

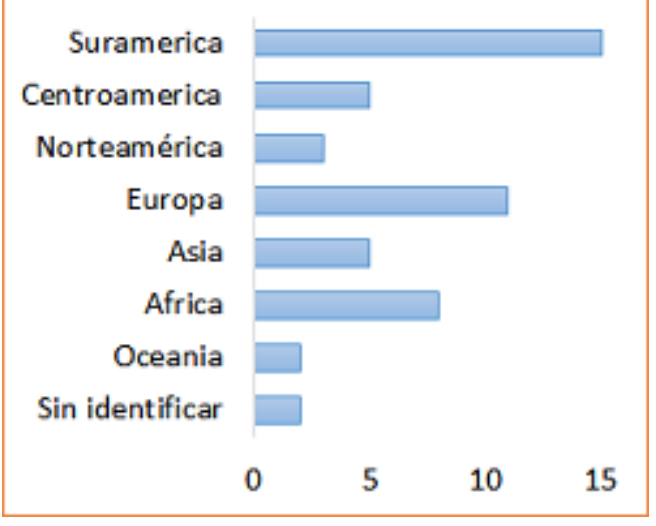

Gráfica 2. Cantidad de artículos científicos por origen de investigación.

En la búsqueda de información en las diferentes bases de datos (ver gráfica 1), se indica en la base de datos de ScieELO.org que existen 529 artículos, de los cuales solo 10 hacen referencia a la enseñanza de las ciencias y 7 específicamente a Biología, se aclara que hubo muy pocos artículos que responden a los componentes de búsqueda "enseñanza y docencia en general, "enseñanza de las ciencias", "enseñanza de la ciencia escolar" y "formación de profesores de Biología" cuando se usa el operador AND con la palabra TIC (y todas sus relaciones de enlace). En el caso de los 184 artículos de sciencedirect.com, donde se emplearon los mismos componentes de búsqueda, se 


\section{Bio - grafía. Escritos sobre la Biología y su Enseñanza. ISSN 2027-1034}

\section{Edición Extraordinaria. p.p. 1149 - 1156}

Memorias del IX Encuentro Nacional de Experiencias en Enseñanza de la Biología y la

Educación Ambiental. IV Congreso Nacional de Investigación en Enseñanza de la Biología.

establece que son muy pocos referentes encontrados ( 9 artículos relevantes para la enseñanza de las ciencias y 7 para Biología). Teniendo en cuanta la misma estrategia de búsqueda se identificaron en ojs.pensamultimedia (33 artículos) 8 artículos relevantes y 3 específicamente a Biología. En el caso de oei.es (18 artículos) 4 están relacionados con la enseñanza y ninguno con Biología. Mientras que en ojs.pensamultimedia se encontraron 6 artículos en la enseñanza de las ciencias y 2 específicamente en Biología de 33 artículos. En cambio, en ries.universia.net (21 artículos) 7 artículos en enseñanza de las ciencias. Y en eduteka.org (60 artículos) 13 artículos hacen referencia a la enseñanza de las ciencias y 9 específicamente Biología.

De los anteriores datos, se determina que 57 artículos específicos permitieron construir la categoría "Uso de TIC" que referencian a la enseñanza a través de TIC. De esta cantidad, se establece que 28 artículos científicos, por su especifidad, contribuyen a consolidar la subcategoría "función de TIC" de los cuales 12 responden a la función de las TIC en la enseñanza de la Biología. Cabe decir que la mayor contribución de artículos científicos entre el periodo 2005 - 2015 por continente (ver gráfica 2), lo realizó Suramérica (15 artículos) seguida de Europa (12 artículos).

\section{DISCUSIONES INTERPRETATIVAS DEL ANÁLISIS DEL CONTENIDO SOBRE FUNCIONES DE LAS TIC.}

La revisión indica que existen funciones asociadas a la cotidianidad del profesor, Kubiatko, (2006) y Tejedor y García (2006), indican dentro de las funciones instrumentales que realizan la mayoría de profesores en su cotidianidad (no relacionadas con la enseñanza o espacio escolar), se encuentran las de poco esfuerzo como: acceso a la internet e información usar los buscadores y enviar y recibir mensajes por correo electrónico (Tejedor \& García 2006). Estos últimos autores indican las funciones instrumentales adquiridas por rutinas, en la mayoría de profesores, responde a: editar documentos de texto y editar tablas e imágenes, y la función que no requiere de esfuerzo consciente es la de manejar ventanas. Según Nechita y Timofti (2011) y Occelli, Garcia y Masullo (2012), los profesores emplean en su mayoría las TIC (internet) para desarrollar la función procedimental de adquirir la información (búsqueda y adquisición de la información). En cuanto la función comunicativa, Occelli, Garcia y Masullo (2012), establece que la mayoría (casi la totalidad) de estos le atribuyen función comunicativa interpersonal a las TIC, siendo el carácter asíncrono (correo electrónico) el predomínate y muy baja el carácter síncrono (chat). Y en Nechita y Timofti (2011), se identifica la función lúdica donde los maestros desarrollan actividades de juegos y relajación.

Para el caso de las "funciones en el uso de TIC en la enseñanza", las primeras funciones identificadas en la enseñanza son las funciones instrumentales. En Afanador (2013) y Skutil (2014), se identifica las funciones instrumentales adquiridas por rutinas de aprendizaje donde las más predominantes son: el registro de notas, diligenciamiento de formularios, presentación de informes, acceso rápido y preparación de batería de ejercicios. Mientras que Paredes y Dias (2012) y Domingo y Marquès (2011), hacen 


\title{
Bio - grafía. Escritos sobre la Biología y su Enseñanza. ISSN 2027-1034
}

\section{Edición Extraordinaria. p.p. 1149 - 1156}

\author{
Memorias del IX Encuentro Nacional de Experiencias en Enseñanza de la Biología y la \\ Educación Ambiental. IV Congreso Nacional de Investigación en Enseñanza de la \\ Biología.
}

referencia a las funciones instrumentales de poco esfuerzo, como: uso de banco de recurso y la exposición magistral y corrección de ejercicios, respectivamente.

Complementariamente a la anterior función se encuentran las funciones procedimentales asignadas a las TIC. Según en Afanador (2013) y Valencia y Said (2014) se identificó en los resultados de profesores la predominancia de la función adquisición de la información (búsqueda, selección y consulta de la información), el cual se realiza por medio del acceso a internet. En los resultados Skutil (2014), existe función interpretación de la información (representación, demostración, y explicación de un tema mediante las TIC). En cambio, la función análisis y realización de inferencias a partir de la información, Nechita y Timofti (2011) indica que es la menos desarrollada en la enseñanza y que tan solo los profesores se limitan a la síntesis. Sin embargo, en la función comprensión de la información, los profesores desarrollaron actividades de enseñanza con TIC como el diseño e implementación de ambientes (Sanabria, López \& Leal, 2014) y construcción de mapas conceptuales (Paredes \& Dias, 2012).

Para la función de gestión se subdividieron según el sitio en el cual se busca desarrollar la acción con el uso de las TIC. En la gestión administrativa Afanador (2013), indica que los profesores usan las TIC en el acompañamiento y seguimiento a procesos institucionales y educativos. Mientras que, en gestión del trabajo en equipo, Afanador (2013), Hernández (2015), Poveda (2014) y Skutil (2014), señalan que los profesores asignan las siguientes funciones favorables de las TIC como: recursos para la cooperación entre pares y/o participación en procesos de construcción colectiva, además indican que las TIC permiten generar espacios de mutua colaboración en redes de apoyo y crear comunidades sobre temas específicos de conocimiento. Y en la función de gestión áulica, Ocampo, Gómez y Zambrano (2015) y Sanabria, López y Leal (2014), destacan que es la que permite realizar la planeación y desarrollo de los diferentes momentos de la clase. Sin embargo, Afanador (2013), indica que las TIC en esta función no están cubriendo la demanda en el seguimiento de convivencia y realización de actividades de recuperación en estudiantes.

Otra de las funciones identificada es la comunicativa en los profesores, las TIC tienen función sincrónica (chat) y asincrónica (foros, correos y mensajes de texto) en los procesos de enseñanza presencial (Afanador, 2013; Domingo \& Marquès, 2011; Hernández, 2015; Valencia \& Said, 2014). Esta función también está relacionada con la interacción social o construcción de red, el cual está creciendo entre los profesores y la misma enseñanza (Afanador, 2013; Valencia \& Said, 2014) expandiéndose por fuera del aula (Paredes y Dias 2012) donde la mayoría de comentarios colectivos de los profesores se realizan a partir de videos o información de internet (Domingo \& Marquès, 2011). Además, la función comunicativa se encuentra relacionada con la baja publicación de contenidos de enseñanza en la web (Valencia \& Said, 2014) y con el alto uso de TIC en la función expositiva, por parte de los profesores en su labor (Akinjide, Adesina \& Osunrinade, 2014).

Desde la enseñanza con TIC, la función de aprendizaje, tienden con mayor frecuencia en los profesores a la realización de actividades demostrativas e ilustrativas a nivel individual o grupales (Nechita \& Timofti, 2011; Paredes \& Dias, 2012; Domingo \& Marquès, 2011; Hernández, 2015). Cabe decir que investigadores como Akinjide, Adesina y Osunrinade 


\section{Bio - grafía. Escritos sobre la Biología y su Enseñanza. ISSN 2027-1034}

\section{Edición Extraordinaria. p.p. 1149 - 1156}

Memorias del IX Encuentro Nacional de Experiencias en Enseñanza de la Biología y la Educación Ambiental. IV Congreso Nacional de Investigación en Enseñanza de la Biología.

(2014), Paredes y Dias (2012) y Skutil (2014) identificaron en los profesores que las TIC cumple la función de facilitar el aprendizaje individual, fortalecer el aprendizaje y construir colabrativamente el conocimiento. Sin embargo, hasta ahora se está incorporando las TIC en la resolución de problemas (Nechita \& Timofti, 2011). Al respecto Poveda (2014), establece dentro de esta función que la información digital (web u otra) debe estar relacionada con los saberes previos y el discurso cotidiano.

En cuento a la función formación en TIC, es importante destacar que los profesores consideran a las TIC recursos importantes para la construcción de experiencias formativas (Hernández, 2015), siendo aspecto fundamental en la formación, la actualización en el uso didáctico de herramientas tecnológicas más que cumplir con requerimientos del ente territorial y Ministerio de Educación Nacional en cuanto a TIC (Afanador, 2013).

\section{CONCLUSIONES}

Según la bibliometría existe baja cantidad de artículos especializados en la enseñanza de la Biología a través de TIC entre el periodo de 2005 - 2015. Esto mismo ocurre en los resultados bibliométricos en el periodo 1997 - 2003, en el área de ciencias y tecnología (Anta \& Pérez 2004), de igual modo Carbonell, Guardiola, Frederic \& Panova, (2016) ratifican que en el periodo 1996 - 2000 la búsqueda de información desde la especificidad tiende hacer baja. Sin embargo, la construcción de la categoría uso de TIC y su subcategoría "funciones de las TIC" contribuye con la productividad científica en el campo de Educación y TIC.

En cuanto al análisis del contenido se establece que las funciones de las TIC que le otorgan los profesores en su cotidianidad (instrumental, procedimental, comunicativa y lúdica) son transferidas o en muchos, a la enseñanza en ciencias o en general, donde el profesor asigna funciones de: acceso y navegación en internet, edición de documentos, adquisición de la información y comunicación sincrónica y asincrónica. Cabe destacar que los profesores asignan funciones específicas a las TIC en su práctica de enseñanza relacionadas con funciones: procedimental (interpretación de la información), comunicativa (migración y participación en redes académicas), aprendizaje (fortalecer la comprensión, motivar la clase, ilustrar conceptos, desarrollar la creatividad y resolución de problemas), de gestión (planeación de clases, desarrollo de nuevas metodologías, crear comunidades sobre temas del conocimiento). Estas particularidades en la práctica de enseñanza de la Biología a través de TIC, cuestionarían si ¿la disponibilidad de los artefactos y la función asignada determinan la integración de las TIC? o ¿la experiencia de integración del profesor de Biología es la que permite disponer de los recursos existentes para otorgarle funciones diferentes 0 complementarias a las funciones primarias de las TIC? 
Bio - grafía. Escritos sobre la Biología y su Enseñanza. ISSN 2027-1034

Edición Extraordinaria. p.p. 1149 - 1156

Memorias del IX Encuentro Nacional de Experiencias en Enseñanza de la Biología y la

Educación Ambiental. IV Congreso Nacional de Investigación en Enseñanza de la

Biología.

\section{REFERENTES BIBLIOGRÁFICOS}

Afanador, H. (2013). Diagnóstico sobre el uso y apropiación de TIC en docentes de la Secretaría de Educación Distrital. XIV Encuentro Internacional Virtual Educa. Medellín, Colombia [En línea http://www.virtualeduca.info/ponencias2013/verponencias.php].

Akinjide, K. Adesina, K. \& Osunrinade, O. (2014). Investigating the Pattern of ICT Utilisation for Science Teaching in Federal Unity Schools in Nigeria Using Kumar's, Subramaniam and Mukherjee Model Approach. International Research: Journal of Library \& Information Science. 4 (2), 305 - 315.

Almerich, C. Suárez, R. Jornet, M. \& Orellana, A. (2010). Las competencias y el uso de las Tecnologías de Información y Comunicación (TIC) por el profesorado: estructura dimensional. Revista electrónica de investigación educativa, 13 (1), $28-42$.

Anta, C. \& Pérez, J. (2004). El uso de las TIC en la educación: Análisis bibliométrico en revistas españolas. II Congreso Online del Obsenvatorio para la CiberSociedad En http://www.cibersociedad.net/congres2004/index es.html"

Carbonell, X. Guardiola, E. Frederic F. \& Panova T. (2016). Trends in Scientific Literature on Addiction to the Internet, Video Games, and Cell Phones from 2006 to 2010. International Journal of Preventive Medicine. 7 (63), IP: 84.88.226.251.

Domingo, M. \& Marquès, P. (2011). Aulas 2.0 y uso de las TIC en la práctica docente. Revista Científica de Educomunicación. 19 (37), 169 - 175.

Escorcia-Oyola, L. y Jaimes de Triviño, C. (2015). Tendencias de uso de las TIC en el contexto escolar a partir de las experiencias de los docentes. Educ. Educ. 18 (1), 137-152. DOI: 10.5294/edu.2015.18.1.8

Hernández, Y. (2015). Factores que favorecen la innovación educativa con el uso de la tecnología: una perspectiva desde el proyecto coKREA. Revista Virtual Universidad Católica del Norte, 45, 38 - 52.

Kubiatko M. (2006). How do teachers use information and communication technology in biology teaching? Information \& communication technology in natural science education 2006, $46-50$.

Nechita, E. \& Timofti, I. (2011). Increasing Independence versus Increasing Collaboration with ICT Support. International Conference on Education and Educational Psychology (ICEEPSY 2011). Procedia - Social and Behavioral Sciences 29, 1508 - 1517.

Niederhauser, D. \& T. Stoddart (2001). Teachers' instrucctional perspectivas and use of educational software. Teaching \& Teacher Education, 17 (1), 15-31. 
Bio - grafía. Escritos sobre la Biología y su Enseñanza. ISSN 2027-1034

Edición Extraordinaria. p.p. 1149 - 1156

Memorias del IX Encuentro Nacional de Experiencias en Enseñanza de la Biología y la Educación Ambiental. IV Congreso Nacional de Investigación en Enseñanza de la Biología.

Ocampo, A. Gómez, M. Zambrano D. (2015). Percepción del profesor sobre el uso del blearning para fortalecer competencias laborales. Revista Innovación Educativa. 7 (2), 1 16.

Occelli, M. Garcia, L. \& Masullo, M. (2012). Integración de las TICs en la formación inicial de docentes y en sus prácticas educativas. Revista fundamentos e investigación, 5, 53 72 .

Paredes, J. \& Dias, R. (2012). La motivación del uso de las TIC en la formación de profesorado en educación ambiental. Ciência \& Educação, 18 (2), 353 - 368.

Sanabria, L. López, O. \& Leal, L. (2014). Desarrollo de competencias metacognitivas e investigativas en docentes en formación mediante la incorporación de tecnologías digitales: aportes a la excelencia docente. Revista Colombiana de Educación. 67, 147170.

Skutil M. (2014). ICT as one of the teaching methods at primary school from a teacher's point of view. Problems of education in the 21st century. 61, 105-112.

Tejedor F. \& García-Valcárcel A. (2006). Competencias de los profesores para el uso de las TIC en la enseñanza. Análisis de sus conocimientos y actitudes. Revista española de pedagogía, 233, 21 - 44.

UNESCO (2013). Enfoques estratégicos de las TICS en educación en América Latina y el Caribe. Santiago de Chile.

Valencia, C. \& Said, H. (2014). Análisis del uso de las TIC como herramientas de enseñanza - aprendizaje en los docentes del Distrito de Barranquilla. Actas 14 del VII Simposio. Las Sociedades ante el Reto Digital 2014. Madrid; asociación de comunicación y nuevas tecnologías, $12,356-366$. 